\title{
Begonia lunaris E. L. JaCques (Begoniaceae), uMA NOVA ESPÉcIe PaRa O ESTAdo do Rio de JANEIRO, BRASIL
}

Eliane de Lima Jacques ${ }^{1}$

\section{Resumo}

(Begonia lunaris E.L.Jacques (Begoniaceae), uma nova espécie para o estado do Rio de Janeiro, Brasil) Uma nova espécie de Begonia, endêmica da mata atlântica do Brasil, é descrita e ilustrada. Begonia lunaris é conhecida somente da sua localidade típica, Estação Ecológica Estadual do Paraíso, localizada nos municípios de

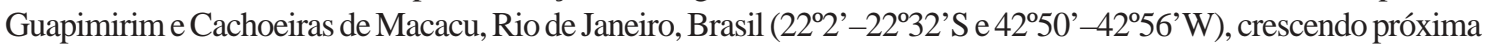
às margens dos rios. Destaca-se prontamente das demais espécies brasileiras do gênero por apresentar alas da cápsula inflexas, flores alaranjadas a vermelho-alaranjadas e folhas semi-lunares. Caracteres diagnósticos, descrição, ilustração detalhada e comentários taxonômicos são fornecidos e suas afinidades são discutidas.

Palavras-chave: taxonomia, mata atlântica, endemismo.

\section{Abstract}

(Begonia lunaris E.L.Jacques (Begoniaceae), a new species from the state of Rio de Janeiro, Brazil) A new narrow endemic species of Begonia from the Atlantic rain forest of Brazil is described and illustrated. Begonia lunaris is known only from the type locality, Estação Ecológica Estadual do Paraíso, localized in the Municipality of

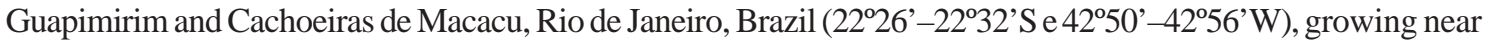
rivers. It promptly differs from the other Brazilian species of the genus in having wings of the capsule bent inwards, orange to orange-red flowers and crescent-shaped leaves. Diagnostic characters, description, detailed illustration and taxonomic comments are given and its relationships are discussed.

Key words: taxonomy, atlantic forest, endemism.

O gênero Begonia possui aproximadamente 1.400 espécies e está amplamente distribuído nas regiões tropicais e subtropicais do mundo (Clement et al. 2004; Forrest et al. 2005; Forrest \& Hollingsworth 2003), ausente apenas na Australásia (Heywood et al. 2007). No Brasil, o gênero está representado por cerca de 200 espécies, distribuído em todas as formações vegetais, excetuando-se os manguezais. Um dos centros de diversidade do gênero é a mata atlântica (Gomes da Silva \& Mamede 2000; Jacques 1996; Jacques \& Mamede 2004; Souza \& Lorenzi 2005).

Begonia possui uma ampla variabilidade morfológica (Jacques \& Mamede 2005) e pode ser caracterizado pelas folhas assimétricas, flores estaminadas com quatro tépalas e pistiladas com cinco, ovário ínfero, placentação axilar, placenta inteira ou partida e cápsulas trialadas.

A possibilidade do uso da subdivisão da placenta, inteira ou partida, na classificação do gênero em seções é questionada por diversos autores (De Candolle 1861; Doorenbos et al. 1998; Irmscher 1925; Jacques 2002; Smith \& Schubert
1946; Warburg 1894). Entretanto, o uso desta característica tem um importante papel na identificação das espécies brasileiras. Das 55 espécies brasileiras de Begonia com placenta partida, 48 ocorrem na mata atlântica.

Begonia lunaris E.L.Jacques, sp. nov. Tipo: BRASIL. RIO DE JANEIRO: Cachoeiras de Macacu, Estação Ecológica Estadual do Paraíso e Centro de Primatologia do Rio de Janeiro (CPRJ-FEEMA), Rio Paraíso, caminho do encanamento, $22^{\circ} 28^{\prime} 51,2^{\prime \prime} \mathrm{S}-42^{\circ} 54^{\prime} 46,9^{\prime \prime} \mathrm{W}$, 3.IX.2007, E.L. Jacques, L.S. Sylvestre, C.M. Mynssen \& A.V.S. Couto 1670 (Holótipo RBR; Isótipo RB).

Fig. 1

Plantis pilis glanduliferis sparsis ferentibus; foliis basifixis, laminis papyraceis, lunaribus, cimis 35-70-floribus, $8,5-15 \mathrm{~cm}$ longis, 4-5-ramosis; tepalis coccineis, placentis bilamellaribus, laminis adpressis externe solum ovuliferis, stigmatibus bifidis; capsulis ellipticis, alis aequalibus, lunaribus, inflexis. A B. maculata foliis lunaribus (non ellipticis),

Artigo recebido em 11/2007. Aceito para publicação em 01/2008.

${ }^{1}$ Universidade Federal Rural do Rio de Janeiro, Instituto de Biologia, Departamento de Botânica, BR-465, km 7, 23890-000, Seropédica, RJ, Brasil. ejacques@ufrrj.br; ejacques@click21.com.br 
capsulis ellipticis (non cordiformis), alis inflexis (non planis), prophyllis persistentibus (non caducis) differt.

Ervas a subarbustos eretos, rupícolas a saxícolas, umbrófilas, 0,5-1,1 m alt., tricomas glandulares esparsos, hialinos no material vivo, vermelhos no material herborizado. Caule ereto, entrenós (1-)3-5(-7,5) cm compr., glabros, nós ligeiramente mais espessados, castanhos na porção proximal, verdes na distal. Pecíolo $3-$ $9,4 \mathrm{~cm}$ compr., cilíndrico, verde-claro, às vezes com leve tonalidade rósea, tricomas glandulares esparsos. Estípulas 2,5-3,6 (4,5-6,1) × (1-1,5-) 1,9-2,5 cm, decussadas, caducas, oblongas a triangulares, carnosas, esverdeadas, passando a marrons, tricomas glandulares em ambas as faces, esparsos na face dorsal, glabrescentes na ventral. Folhas (15-)22-34×(6,7-)10-15 cm, assimétricas, semi-lunares, basifixas, papiráceas, discolores, face adaxial verde-escura, lustrosa, com máculas alvas a argênteas, elípticas ou ovaladas, geralmente associadas às folhas jovens, glabra, face abaxial verde-escura, verde-clara a vinácea, com tricomas glandulares esparsos, ápice acuminado, base cordiforme, margem serrulada, nervuras principais $6-8$, proeminentes e verde-claras na face adaxial, nervação actinódroma. Cimeiras 8,5-15 cm compr., pêndulas, 4-5-ramificadas, 35-70 flores; pedúnculo ca. $5 \mathrm{~cm}$ compr., cilíndrico, alaranjado a vermelho-alaranjado. Brácteas 1,7-1,9 cm compr., ovadas a largamente ovadas, carnosas, persistentes, margem inteira, alaranjadas a vermelho-alaranjadas. Flores estaminadas 3$3,5 \mathrm{~cm}$ compr., vistosas, levemente aromáticas, alaranjadas a vermelho-alaranjadas, pedicelo (1,5-)2,4-3,5 cm compr., tépalas-4, externas (12-)21-25×(14-)22-25 mm, ovadas a largamente ovadas, tricomas glandulares esparsos, internas 11-16×5-6 mm, elípticas a obovadas, glabras, estames 56-65(-80), 4-6 mm compr., amarelos, filetes $1-2 \mathrm{~mm}$ compr., livres, anteras $1-1,5 \mathrm{~mm}$ compr., obovadas, recurvadas, extrorsas, conectivo alargado. Flores pistiladas 4-4,5 cm compr., vistosas, levemente aromáticas, alaranjadas a vermelho-alaranjadas, pedicelo $1-1,5 \mathrm{~cm}$ compr.; tépalas-5, desiguais, 4 maiores, (10-)19-24× (6-8) 15-20 mm, ovadas a largamente ovadas e 1 menor, (7-)12-18 $\times(3-) 5-11 \mathrm{~mm}$, obovadas a espatuladas, margem inteira, tricomas glandulares esparsos, profilos-2, (6-)12-16× 9-16 mm, largamente ovados, margem inteira, persistentes, estiletes bifurcados, (3-) 6-7 mm compr., espiralados, cilíndricos, base flabeliforme, amarelos, estigmas-3, papilas estigmáticas dispostas nas margens dos ramos, ovário trilocular, 22-29 $\times 15 \mathrm{~mm}$, oblongo, alaranjado a vermelho-alaranjado, tricomas glandulares esparsos, alas ligeiramente inflexas, placenta bipartida, lamelas adpressas, óvulos na face externa. Cápsulas trialadas, $2-2,7 \times 1,5-1,8 \mathrm{~cm}$, oblongas, pêndulas, quando imaturas com tricomas glandulares esparsos, pedúnculo ca. $1,5 \mathrm{~cm}$ compr., alas $6-12 \times 6-12 \mathrm{~mm}$, semelhantes entre si, lunadas, inflexas, profilos persistentes, basais. Sementes oblongas.

Parátipos: BRASIL. RIO DE JANEIRO: Estação Ecológica Estadual do Paraíso, Centro de Primatologia do Rio de Janeiro: Cachoeiras de Macacu, afluente do rio Anil, 5.IV.1976, fr., D. Araújo et al. 1068 (GUA); Rio Paraíso, $22^{\circ} 00^{\prime} S-42^{\circ} 03^{\prime} \mathrm{W}$, às margens do rio Falcão, trilha para o Morro do Pirulito, vale do rio Falcão, 22.XI.1991, fl. estaminada ${ }_{2}$ L. Sylvestre et al. 689 (RB); 22030'S-42053'W, 5.II.1992, fl. estaminada, C. M. Vieira 148 (RB); ibidem, 6.II.1992, fl. pistilada efr., C. M. Vieira 159 (RB); Rio Paraíso, próximo à represa da CEDAE, 26.III.1992, fl. pistilada e fr., T. Fontoura et al. 274(RB); caminho do encanamento, $22^{\circ} 28^{\prime} 55,6^{\prime \prime} \mathrm{S}-42^{\circ} 54^{\prime} 48,9^{\prime \prime} \mathrm{W}$, 3.IX.2007, fr., E. L. Jacques et al. 1671 (RBR); Guapimirim, $150 \mathrm{~m} \mathrm{s.m.,} \mathrm{22.XI.1984,} \mathrm{fl.} \mathrm{estaminada} \mathrm{efr.,}$ G. Martinelli et al. 10375 (RB); trilha da casa em ruínas, 2.II.2006, fr., C. G. Campos et al. 60 (HB); ibidem, 2.XI.2006, fl. estaminada, J. R. A.Oliveira et al. 41 (HB). Distribuição, habitt e fenologia: Begonia lunaris é conhecida somente da localidade típica, Estação Ecológica Estadual do Paraíso. Esta Unidade de Conservação (UC) estende-se nos municípios de Guapimirim e Cachoeiras de Macacu, Rio de Janeiro, e situa-se na vertente oceânica da Serra do Mar, denominada Serra dos Órgãos. Na sede desta UC, localizada no município de Guapimirim, funciona o Centro de Primatologia do Rio de Janeiro - CPRJ-FEEMA. $B$. lunaris cresce em floresta baixo montana, em pequenas e esparsas populações, sobre ou entre as rochas, à margem dos rios ou no interior da floresta, em semi-sombra. Foi coletada com 


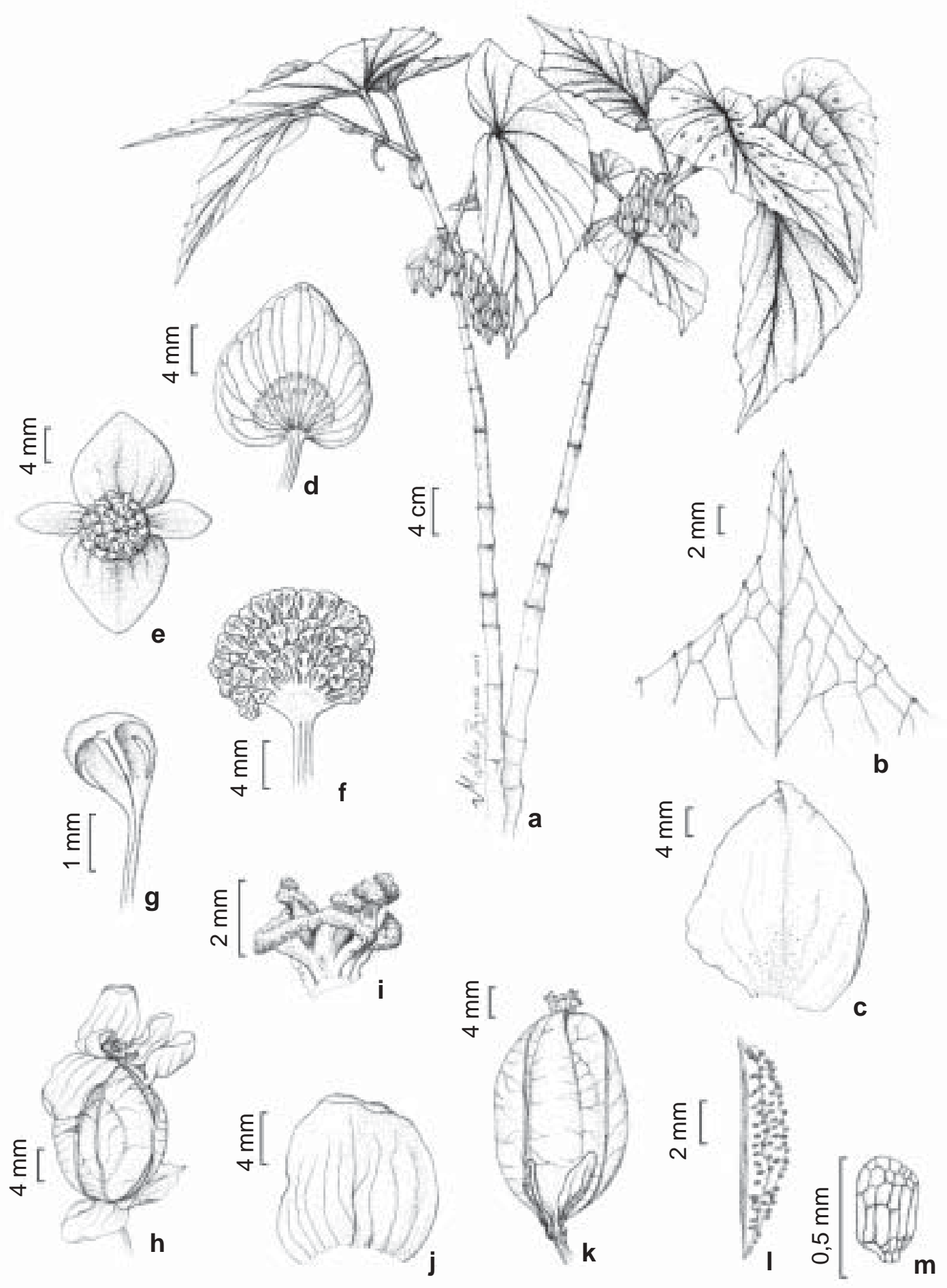

Figura 1 - Begonia lunaris E.L. Jacques - a. hábitos; b. ápice foliar; c. estípula; d. botão floral (flor estaminada); e. flor estaminada; f. androceu; g. estame (vista ventral); h. flor pistilada (reidratada); i. estiletes; j. profilo da flor pistilada; $\mathrm{k}$. fruto com profilos e estiletes persistentes; 1. placenta (retirada do fruto); m. semente. 
flores e frutos entre os meses de setembro a abril. As flores são levemente aromáticas, com perfume de rosas e alaranjadas a vermelhoalaranjadas. Esta última característica, muito rara nas espécies brasileiras, evidencia o potencial ornamental desta espécie. Uma vez que o táxon possui uma área de ocorrência estimada em menos de $100 \mathrm{~km}^{2}$, é conhecido de uma única localidade e a maioria dos indivíduos observados é encontrada em subpopulações pequenas e relativamente isoladas, $B$. lunaris é reconhecida na categoria de Criticamente em Perigo (CR), de acordo com os critérios da União Mundial para a Conservação da Natureza (IUCN 2001: B1a).

Begonia lunaris pode ser facilmente reconhecida pelas alas da cápsula inflexas, flores alaranjadas a vermelho-alaranjadas e folhas semi-lunares. Dentre as 55 espécies brasileiras de Begonia com placenta partida, B. lunaris assemelha-se $B$. maculata Raddi e $B$. undulata Schott pelo hábito ereto, lamelas da placenta adpressas, anteras obovadas, flores pistiladas com dois profilos e cápsulas com alas semelhantes entre si. Entretanto, B. maculata e B. undulata diferem de $B$. lunaris pelas cápsulas cordiformes (vs. oblongas), com alas planas (vs. inflexas) e profilos caducos ( $v s$. persistentes). B. maculata possui folhas assimétricas, transversalmente elípticas, 13,5-20 ×4,5-6 cm (vs. 15-34 ×6,7$15 \mathrm{~cm}$ ) e $B$. undulata folhas simétricas, ovadas a lanceoladas, $8-13 \times 1,5-3,9 \mathrm{~cm}$.

\section{Agradecimentos}

A Maria Alice Rezende pelo preparo das ilustrações. Aos curadores dos herbários GUA, $\mathrm{HB}$ e RB que permitiram o estudo de suas coleções. A Marcos Peron pela entrega de dois slides que me alertaram para a descoberta da nova espécie. A Lana da Silva Silvestre, Claudine M. Mynssen e Arthur Vinícius dos Santos Couto que gentilmente me acompanharam na coleta do material-tipo. Ao chefe da Estação Ecológica Estadual do Paraíso e Centro de Primatologia do Rio de Janeiro (CPRJ-FEEMA), pela autorização de coleta na área. Ao Instituto de Pesquisas do Jardim Botânico do Rio de Janeiro pela concessão do veículo para a coleta do material-tipo. À minha filha, Isadora, pela sugestão do nome da espécie (begônia meia-lua).

\section{REFERÊNCIAS BIBLIOGRAFIA}

Clement, W. L.; Tebbit, M. C.; Forrest, L. L.; Blair, J. E.; Brouillet, L.; Eriksson, T. \& Swensen, S. M. 2004. Phylogenetic position and biogeography of Hillebrandia sandwicensis (Begoniaceae): a rare Hawaiian relict. American Journal of Botany 91(6): 905-917.

De Candolle, A. 1861. Begoniaceae. In: Martius, C. P. F. (ed.). Flora brasiliensis. F. Fleischer, Lipsiae 3(1): 338-396.

Doorenbos, J.; Sosef, M. S. M. \& De Wilde, J. J. F. E. 1998. The sections of Begonia. Wageningen Agricultural University Papers 98: 1-266.

Forrest, L. L. \& Hollingsworth, P. M. 2003. A recircumscription of Begonia based on nuclear ribosomal sequences. Plant Systematic and Evolution 241: 193-211.

Forrest, L. L.; Hughes, M. \& Hollingsworth, P. M. 2005. A phylogeny of Begonia using nuclear ribosomal sequence data and morphological characters. Systematic of Botany 30(3): 671-682.

Gomes da Silva, S. J. \& Mamede, M. C. H. 2000. A new species of Begonia (Begoniaceae) from the Atlantic Coastal Forest in the State of São Paulo, Brazil. Novon 10: 22-25.

Heywood, V. H.; Brummitt, R. K.; Culham, A. \& Seberg, O. 2007. Flowering plant families of the world. Firefly Books, Ontario, 424p.

Irmscher, E. 1925. Begoniaceae. In: Engler, A. \& Prantl, K. (eds.). Die natürlichen Pflanzenfamilien. 2ed. Vol. 21. Pp. 548-588.

IUCN. 2001. IUCN Red List Categories and Criteria: Version 3.1. IUCN Species Survival Commission. IUCN, Gland, Switzerland, and Cambridge, UK, 30 pp.

Jacques, E. L. 1996. Begoniaceae. In: Lima, M. P. M. \& Guedes-Bruni, R. R. (eds.). Reserva Ecológica de Macaé de Cima, Nova Friburgo, RJ - Aspectos florísticos das espécies vasculares. Vol. 2. Jardim Botânico do Rio de Janeiro, Rio de Janeiro. Pp. 93-133. 
2002. Estudos taxonômicos das espécies brasileiras do gênero Begonia L. (Begoniaceae) com placenta partida. Tese de Doutorado. Universidade de São Paulo, São Paulo, 319p.

Jacques, E. L. \& Mamede, M. C. H. 2004. Novelties in Begonia (Begoniaceae) from the coastal forests of Brazil. Brittonia 56(1): 75-81.

2005. Notas nomenclaturais em Begonia L. (Begoniaceae). Revista Brasileira de Botânica 28: 579-588.
Smith, L. B. \& Schubert, B. G. 1946. The Begoniaceae of Colombia. Caldasia 4: 3 38, 77-107, 179-209.

Souza, V. C. \& Lorenzi, H. 2005. Botânica sistemática: guia ilustrado para identificação das famílias de angiospermas da flora brasileira, baseado em APG II. Instituto Plantarum, Nova Odessa, 640p.

Warburg, O. 1894. Begoniaceae. In: Engler, A. \& Prantl, K. (eds.). Die natürlichen Pflanzenfamilien. Aufl. Leipzig (Wilhelm Engelmann). 3(6a): 121-150. 